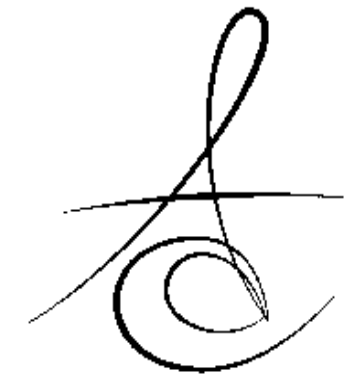

\section{LİTYUM DİSİLİKAT İLE GÜÇLENDİRİLMİŞ CAM SERAMİKLER}

\section{LITHIUM DISILICATE GLASS CERAMICS WITH REINFORCED}

\author{
Arş. Gör. Dt. Alper ÖZDOĞAN**
}

Makale Kodu/Article code: 2256

Makale Gönderilme tarihi: 27.04.2015

Kabul Tarihi: 20.08.2015

\section{ÖZET}

Diş hekimliği pratik uygulamalarında yapılacak olan restorasyonun klinik başarısı seçilen materyalin türüne bağlı olarak değişebilmektedir. Estetiğin ve fonksiyonun önemli olduğu günümüzde, tam seramik sistemler metal destekli seramiklere göre daha başarıı sonuçlar vermektedir. Tam seramik sistemler grubunda yer alan lityum disilikat ile güçlendirilmiş cam seramikler ise sahip olduğu üstün estetik özelliklerinden dolayı diğer seramiklere nazaran daha ön planda yer almaktadır. $\mathrm{Bu}$ derlemede, lityum disilikat ile güçlendirilmiş cam seramik sistemler hakkında bilgi verilmiştir.

Anahtar Kelimeler: Lityum disilikat, seramik, estetik.

\section{ABSTRACT}

In the clinical practice of dentistry, the success of the restoration can be changed to type of the material. Nowadays, esthetic and function is importants of full ceramic systems give more success ful results than the metal ceramic systems. Lithium disilicate glass ceramics which located in the group of full ceramic systems, are situated in the fore ground of the other ceramics due to the superior esthetic properties. In this review, information about the reinforced glassceramic systems are given with lithium disilicate.

Key Words: Lithium disilicate, ceramic, aesthetic.

\section{GİRİ̧̧}

Estetiğe olan ilginin giderek artması, diş hekimliğinde de daha üstün estetik özelliklere sahip yeni materyallerin geliştirilmesine neden olmuştur.

Land $^{1}$ tarafindan 1886 yılında üretilen porselen jaket kuron ilk tam seramik kuron olarak bilinmektedir. Mc Lean'ın 1965 ylında geliştirdiği alümina ile güçlendirilmiş porselen jaket kuronlar, tam seramik kuronlarla ilgili ilerleyen yıllarda yapılacak olan çalışmaların temelini oluşturmaktadır. ${ }^{2}$ Horn, ${ }^{3}$ Simonsen ve Calamia $^{4}$ ve Calamia'nın ${ }^{5} 1983$ yllında, asit-etch ile mineye bağlanan seramikleri piyasaya sürmeleri, 1990'ların başlarında ise dentin adezivlerinin üretilmesi daha yüksek bağlantı direncine sahip restorasyonların yapılmasına yol açmıştır. Bunun sonucu olarak da hekimler, metal seramik sistemlere alternatif bonded seramik sistemleri kullanmaya başlamışlardır. ${ }^{6}$

Tam seramik sistemler, yapım tekniklerine ve kor yapılarına göre iki farklı şekilde sınılandırılabilir. Yapım tekniklerine göre tam seramik sistemler: ${ }^{7,8}$
1. Isıya dayanıklı day'lar üzerinde fırınlanan seramik sistemler

- Aluminus porselenler

- Magnezyum kor

- Cerestore/Alceram (InnotekDentalCorp., USA)

- Optec (Jeneric, PentronInc., USA)

- Hi-Ceram (Vita-Zahn-fabric, Germany)

- In-Ceram (Vita-Zahn-fabric, Germany)

2. Dökülebilir (cam) porselen sistemler

- Dicor (DentsplyCeramco, York, USA)

- Cerapearl (Kyocera, USA)

3. Sıkıştırılabilir porselen sistemler

- IPS Empress (Ivoclar, Schaan, Liechtenstein)

- IPS Empress II (Ivoclar, Schaan, Liechtenstein)

- Finesse AllCeramic (Dentsply, York, USA)

- IPS e.maxPress (Ivoclar, Schaan, Liechtenstein)

- Creationn Press-ceramics (CreationWilliGeller, Meiningen, Austria)

\footnotetext{
* Recep Tayyip Erdoğan Üniversitesi Diş Hekimliği Fakültesi Protetik Diş Tedavisi AD

** Atatürk Üniversitesi Diş Hekimliği Fakültesi Protetik Diş Tedavisi AD
} 
4. CAD/CAM sistemler

- Cerec (SironaDental Siemens, Germany)

- Celay (Mikrona, Germany)

- Procera All-Ceram (Nobel Biocare AB, Göteborg, Sweden)

- Cercon (DeguDent, Germany)

- Precident (DCS Dental AG, Allschwil, Switzerland)

- Lava (3M ESPE, St. Paul, Minn, USA)

- Everest (KavoDental, Biberach, Germany)

- ZenoTech (Wieland, Pforzheim, Germany) Kor yapılarına göre tam seramiklerin sınıflandırılması: ${ }^{9}$

1. Cam seramikler

a. Lityum disilikat

- IPS Empress II (IvoclarVivadent, Schaan, Liectenstein)

- IPS e.max Press (IvoclarVivadent, Schaan, Liectenstein)

b. Lösit

- IPS Empress (IvoclarVivadent, Schaan, Liectenstein)

- Optimal Pressable Ceramic (JenericPentron, Wallingford, Conn)

- IPS Pro CAD (IvoclarVivadent, Schaan, Liectenstein)

c. Feldspatik

- VITABLOCKS Mark II (VITA Zahnfabrik, BadSackingen, Germany)

- VITA Triluxe Bloc (VITA Zahnfabrik, BadSackingen, Germany)

- VITABLOCKS Esthetic Line (VITA Zahnfabrik, BadSackingen, Germany)

2. Alümina seramikler

- In-Ceram Alumina (VITA Zahnfabrik, BadSackingen, Germany)

- In-Ceram Spinell (VITA Zahnfabrik, BadSackingen, Germany)

- Synthoceram (CICERO DentalSystems, Hoorn, Netherlands)

- In Ceram Zirconia (VITA Zahnfabrik, BadSackingen, Germany)

- Procera (Nobel Biocare AB, Göteborg, Sweden)

3. Zirkonya seramikler

- Lava (3M ESPE, St. Paul, Minn)

- Cercon (DentsplyCearmco, York, USA)

- DC-Zirkon (DCS Dental AG, Skelleftea, Sweden)

- Procera (Nobel Biocare AB, Göteborg, Sweden)

\section{Lityum Disilikat ile Güçlendirilmiş Cam Seramikler}

\%57-80 kuartz, \%11-19 lityum oksit ve \%0-5 oranında alüminyum oksitten oluşan seramiklerdir. Materyalin \%60'ını oluşturan, kristal partiküllerinin yoğun ve homojen birbirine bağlanmış yapısı sayesinde, bükülme direnci 320-450 MPa'ya kadar yükseltilmiştir. $^{10}$

Restorasyonun kor yapısı kayıp mum tekniği ile elde edildikten sonra lityum disilikat cam porselen tabletleri viskoz akma özelliğine ulaştırılmakta ve basınçla revetman boşluğunun içine yollanmaktadır. Lityum disilikat seramik kor yapıların hazırlanmasında kullanılan diğer bir yöntem de hazır blokların bilgisayar kontrollü makine ile şekillendirilmesidir. Daha sonra elde edilen kor yapı üzerine, tabakalama tekniği ile florapatit yapıda cam seramik uygulanmaktadır. ${ }^{7}$

Tam seramik restorasyonların kullanım alanını genişletebilmek ve onların köprü yapımında da kullanımını sağlamak amacıyla geliştirilmişlerdir. Lityum disilikat cam seramiklerde ISI ve basınç tekniğinin kullanımının lityum disilikat fazda homojen yapı oluşumunu sağladığı, kontrol edilemeyen mikro çatlakları engellediği, kısa sürede ve kolay restorasyon hazırlanmasına olanak verdiği ifade edilmiştir. ${ }^{11}$

Cam seramikler, cam içinde büyüyen ve çekirdekleşen kristal fazın doğasına bağlı olarak geniş optik-mekanik özelliklerde çeşitli kompozisyonlarda üretilebilirler. $\mathrm{Li}_{2} \mathrm{O}-\mathrm{Al}_{2} \mathrm{O}_{3}-\mathrm{CaO}-\mathrm{SiO}_{2}$ sistemi günümüzde araştırmalarda yaygın olarak kullanılmaktadır. Uygun katkı maddelerinin kullanımı daha sert ve dayanıklı cam seramiklerin geliştirilmesinde kritik öneme sahiptir. $^{12}$

Günümüzde kullanılan lityum disilikatın (\%5780 SiO2, \%0-5 Al2O3,\%0.1-6 La2O3, \%0-5 MgO, \%0$8 \mathrm{ZnO}, \% 0-13$ K2O, \%11-19 Li2O, \%0.5-11 P2O5, \%0-6 katkı maddeleri ve renk pigmentleri) kimyasal yapısı 1998 yılında Schweiger ve Arridge ${ }^{13}$ tarafından açıklanmıştır. Holand ve arkadaşları, ${ }^{14}$ lityum disilikat cam seramiklerin uzamış lityum orto fosfattan oluştuğunu, cam matriksin ise her iki kristal fazı çevrelediğini belirtmişlerdir.

\section{IPS Empress II}

Lityum disilikat içerikli cam seramik olan IPS Empress II (IvoclarVivadent, Schaan, Liechtenstein) 1998 yılında geliştirilmiştir. IPS Empress II lityum disilikattan ve lityum orto fosfattan oluşan bir cam seramiktir. Bu materyal lösit ile güçlendirilmiş IPS 
Empress materyalinden daha dayanıkıdır ve yüksek translüsentliğinden kaynaklanan mükemmel estetiğe sahiptir. Laboratuvar tekniğinin IPS Empress ile aynı olmasına karşın bitimi sadece layering tekniği ile yapilır. ${ }^{15}$

Materyalin içindeki kristaller kırığın ilerlemesini engellediğinden esneme dayanımı oldukça yüksektir. Karşıt doğal diş üzerinde aşındırııı etkisinin az, optik özelliklerinin ve ışık geçirgenliğinin iyi olması nedeniyle klinik olarak geniş kullanım alanı bulmuşlardır. 14, 16-19

IPS Empress II sistemi anterior ve posterior tek kuronlarda, anterior ve posterior üç üyeli köprü yapımında kullanılabilir. Posterior üç üyeli köprülerde kullanılabilmesi için ikinci premolarların en son distal destek olması ve gövdenin bir premolar genişliğinde (yaklaşık 7-8 mm) hazırlanması gerekmektedir. Materyalin kor kısmındaki kimyasal yapı IPS Empress ve IPS Empress II'nin farklılığını oluşturmaktadır. Bu kor yapıdaki farklıık IPS Empress II'nin kırılmaya karşı olan direncini IPS Empress'e göre üç kat artırmıştır. Ayrıca IPS Empress II'de cam daha az olduğu için kırılmaya karşı direnci fazla, mikro çatlak oluşma riski ise en azdır. ${ }^{14,20,21}$ Bükülme dayanımı 300-440 MPa ve kırılma doygunluğu 2,8-3,5 MPa m ${ }^{1 / 2}$ dir. $^{22,23}$

Fradeani ve Redemagni, ${ }^{24} 125$ adet IPS Empress II tek kuronların 11 yıllık klinik takibini yaptıkları çalışmalarında $\% 95,2$ oranında başarı bildirmişlerdir. Marquardt ve Strub da ${ }^{20}$ çalışmalarında 50 aylık klinik kullanım sonucunda IPS Empress II tek kuronlar için $\% 100$, üç üye köprüler için $\% 70$ 'lik başarı oranı belirtmişlerdir.

IPS e.max Press

Ivoclar Vivadent firması, IPS Empress sisteminden sonra, 2005 yilında güçlendirilmiş preslenebilir cam seramik materyali olan IPS e.max Press sistemini piyasaya sürmüştür. Sistemin içeriğinin Empress II sistemi gibi lityum disilikattan oluşmasına karşın, kullanılan farklı firınlama tekniği sayesinde fiziksel özellikleri ve estetiği arttııımışır. ${ }^{25}$

Pres tekniği ile kullanılan lityum disilikat cam seramiktir. Temel kristal faz olan lityum disilikat 3-6 $\mu \mathrm{m}$ uzunluğundaki iğne benzeri kristallerden oluşmaktadır. Lityum disilikat kristaller cam matriks içine gömülmüş şekildedir. IPS e.max Press seramik ingotlar içeriğinde ağırııça; \%57-80 $\mathrm{SiO}_{2}, \% 11-19 \mathrm{Li}_{2} \mathrm{O}, \& 0-13$ $\mathrm{K}_{2} \mathrm{O}, \% 0-11 \mathrm{P}_{2} \mathrm{O}_{5}, \% 0-8 \mathrm{ZrO}_{2}, \% 0-8 \mathrm{ZnO}$ ve $\% 0-10$ diğer oksitler ve seramik pigmentler bulunmaktadır. $\mathrm{Bu}$ teknikte, renk pigmentleri erime ısısına ulaşıldığında eriyeceği için materyale ilave edilmezler. Renk pigmentleri yerine cam içinde çözünen polivalent iyonlar, arzu edilen rengi sağlamak için kullanılır. İyon esasılı renklendirme mekanizmasının kullanılmasının avantajı, renk salan iyonların materyal içinde homojen bir şekilde dağlabilmesidir. Bunun aksine renk pigmentleri mikro yapıda kusurlara neden olmaktadır. Farklı opasitelerde homojen ingot seçenekleri vardır. IPS e.max Press ingotlar, 400 MPa'lık bükülme direnci ile preslenebilir cam seramikler arasında en yüksek direnci gösterirler. IPS Empress ingotlarla arşılaştıııldığında; IPS e.max Press ingotlar, daha fazla restorasyonun üretilebildiği daha geniş çapta ve hacimdedirler. IPS e.max Press sisteminde farklı translusensi değeri sergileyen 3 farklı ingot seçeneği bulunmaktadır. Bunların düşük translusensi özelliği gösteren ingotlar (IPS e.maxPress LT), 9 A-D ve 4 beyazlatma renginde olup ince veneerlerde kullanılabilmektedir. Orta opasiteli ingotlar (IPS e.maxPress MO), MO0-MO4 renklerindedir ve vital ya da hafif renklenmiş preparasyonlar için ideal alt yapıların hazırlanmasına olanak sağlarlar. Yüksek opasiteli ingotlar (IPS e.max Press HO) ise, opasitelerine bağlı olarak devital, yoğun renklenmiş preparasyonların ya da metal alt yapıların maskelenmesinde kullanılabilirler. ${ }^{25}$

IPS e.max Press sistemi, ince veneerler, parsiyel kuronlar, anterior ve posterior kuronlar, 3 üyeli anterior köprüler, destek diş olarak ikinci premolara kadar uzanan 3 üyeli köprüler, anterior ve posterior bölgelerde implant üstü tek kuronlar, ikinci premolara kadar uzanan implant üstü 3 üyeli köprüler ve teleskop kuronlarda kullanılabilmektedir. ${ }^{25}$

Albakry ve arkadaşlarının ${ }^{22}$ yaptığı bir çalışmada, IPS e.max Press'in biaksiyal bükülme direnci, IPS Empress ve IPS Empress II'den daha yüksek bulunmuştur.

\section{SONUÇ}

Lityum disilikat ile güçlendirilmiş cam seramikler, estetik özellikleri nedeniyle klinikte oldukça fazla kullanılmaktadır. Ancak mekanik özelliklerinin yetersizliğine bağlı olarak posterior bölgede kullanımları kısıtıdır. Endikasyon alanının genişletilebilmesi için mekanik ve fiziksel özelliklerinin geliştirilmesi gerekmektedir. 


\section{KAYNAKLAR}

1. Land $\mathrm{CH}$. A new system of restoring badly decayed teeth by means of an enamelled coating. Independent Pract 1886;7:407-9.

2. Jones DW, Wilson HJ. Some properties of dental ceramics. J Oral Rehabil 1975; 2: 379-96.

3. Horn HR. A new lamination: porcelain bonded to enamel. N Y State Dent J 1983; 49: 401-3.

4. Simonsen RJ, Calamia JR. Tensile bond strengths of etched porcelain. J Dent Res 1983;62: 279-90.

5. Calamia JR. Etched porcelain facial veneers: a new treatment modality based on scientific and clinical evidence. N Y J Dent1983; 53: 255-9.

6. Nakabayashi N, Nakamura M, Yasuda N. Hybrid layer as a dentin-bonding mechanism. J Esthet Dent 1991; 3: 133-8.

7. Yavuzyılmaz H, Turhan B, Bavbek B, Kurt E. Tam porselen sistemleri II. Gazi Üniv Diş Hek Fak Derg 2005;22:49-60.

8. Bayındır F, Uzun İH. Tam seramik kuron sistemleri. Atatürk Üniv Diş Hek Fak Derg 2007; Suppl 2: 3342.

9. Conrad HJ, Seong WJ, Pesun IJ. Current ceramic materials and systems with clinical recommendations: a systematic review. J Prosthet Dent 2007; 98: 389-404.

10. Fons-Font A, Sola-Ruiz MF, Granell-Ruiz M, LabaigRueda C, Martinez-Gonzalez A. Choice of ceramic for use in treatments with porcelain laminate veneers. Med Oral Patol Oral Cir Bucal 2006; 11: 297-302.

11. Oh SC, Dong JK, Luthy H, Scharer P. Strength and microstructure of IPS Empress 2 glass-ceramic after different treatments. Int J Prosthodont 2000; 13: 468-72.

12. Anusavice $\mathrm{KJ}$, Zhang $\mathrm{NZ}$, Moorhead JE. Influence of colorants on crystallization and mechanical properties of lithia-based glass-ceramics. Dent Mater 1994; 10: 141-6.

13. Schweiger M, Arridge SR. Comparison of two- and three-dimensional reconstruction methods in optical tomography. Appl Opt 1998; 37: 7419-28.

14. Holand W, Schweiger M, Frank M, Rheinberger V. A comparison of the microstructure and properties of the IPS Empress 2 and the IPS Empress glassceramics. J Biomed Mater Res 2000; 53: 297-303.
15. Uctasli S, Wilson HJ, Unterbrink G, Zaimoglu A. The strength of a heat-pressed all-ceramic restorative material. J Oral Rehabil 1996; 23: 25761.

16. Oktay HG. Tüm Seramik Sistemler, EÜ Dişhek Fak Derg 2003; 24: 11-6.

17. Devlin H. Operative Dentistry. Springer-Verlag Berlin Heidelberg 2006; 85-92.

18. Hooshmand T, Parvizi S, Keshvad A. Effect of surface acid etching on the biaxial flexural strength of two hot-pressed glass ceramics. J Prosthodont 2008;17: 415-9.

19. Al-Wahadni AM, Hussey DL, Grey N, Hatamleh MM. Fracture resistance of aluminium oxide and lithium disilicate-based crowns using different luting cements: an in vitro study. J Contemp Dent Pract 2009; 10: 51-8.

20. Marquardt $P$, Strub JR. Survival rates of IPS empress 2 all-ceramic crowns and fixed partial dentures: results of a 5-year prospective clinical study. Quintessence Int 2006; 37: 253-9.

21. Pilathadka S, Vahalova D. Contemporary allceramic materials, part-1. Acta Medica (Hradec Kralove) 2007; 50: 101-4.

22. Albakry M, Guazzato M, Swain MV. Biaxial flexural strength, elastic moduli, and x-ray diffraction characterization of three pressable all-ceramic materials. J Prosthet Dent 2003; 89: 374-80.

23. Heydecke G, Butz F, Binder JR, Strub JR. Material characteristics of a novel shrinkage-free $\mathrm{ZrSiO}(4)$ ceramic for the fabrication of posterior crowns. Dent Mater 2007; 23: 785-91.

24. Fradeani M, Redemagni M. An 11-year clinical evaluation of leucite-reinforced glass-ceramic crowns: a retrospective study. Quintessence Int 2002; 33: 503-10.

25. Ivoclar Vivadent AG. The Compatible All-Ceramic System, Dental Technician and Instructor, Schaan, Liechtenstein. 2005.
Yazışma Adresi
Prof. Dr. Zeynep YEŞİL DUYMUŞ
Recep Tayyip Erdoğan Üniversitesi
Diş Hekimliği Fakültesi
Protetik Diş Tedavisi Ana Bilim Dalı, Rize
Tel: 064-2220002
E Mail: zyesilz@hotmail.com 